\title{
Rational design of microRNA-siRNA chimeras for multifunctional target suppression
}

\author{
ZHOU JIANG, ${ }^{1,2,3}$ WEIJUN LIU, ${ }^{1,3}$ YUHUI WANG, ${ }^{1,2}$ ZHEN GAO, $^{1}$ GE GAO, ${ }^{1}$ and XIAOWEI WANG ${ }^{1,4}$ \\ ${ }^{1}$ Department of Radiation Oncology, Washington University School of Medicine, St. Louis, Missouri 63108, USA \\ ${ }^{2}$ Health Ministry Key Laboratory of Chronobiology, College of Basic Medicine and Forensic Medicine, Sichuan University, Chengdu, \\ Sichuan 610041, China
}

\begin{abstract}
MicroRNAs (miRNAs) are involved in a variety of human diseases by simultaneously suppressing many gene targets. Thus, the therapeutic value of miRNAs has been intensely studied. However, there are potential limitations with miRNA-based therapeutics such as a relatively moderate impact on gene target regulation and cellular phenotypic control. To address these issues, we proposed to design new chimeric small RNAs (aiRNAs) by incorporating sequences from both miRNAs and siRNAs. These aiRNAs not only inherited functions from natural miRNAs, but also gained new functions of gene knockdown in an siRNA-like fashion. The improved efficacy of multifunctional aiRNAs was demonstrated in our study by design and testing of an aiRNA that inherited the functions of both miR-200a and an AKT1-targeting siRNA for simultaneous suppression of cancer cell motility and proliferation. The general principles of aiRNA design were further validated by engineering new aiRNAs mimicking another miRNA, miR-9. By regulating multiple cellular functions, aiRNAs could be used as an improved tool over miRNAs to target disease-related genes, thus alleviating our dependency on a limited number of miRNAs for the development of RNAi-based therapeutics.
\end{abstract}

Keywords: microRNA; siRNA; RNA interference; target regulation; cancer therapy

\section{INTRODUCTION}

RNA interference (RNAi) is an RNA-guided gene silencing process within living cells that controls the expression of the targeted genes (Hannon 2002; Denli and Hannon 2003; Sontheimer 2005). There are two major types of small RNA molecules that are central to RNA interference: microRNAs (miRNAs) and small interfering RNAs (siRNAs).

miRNAs are a family of small noncoding RNA molecules ( $\sim 22$ nucleotides, nt) that down-regulate the expression of their gene targets (Miska 2005). More than 2000 human miRNAs have been identified to date (Kozomara and Griffiths-Jones 2011). Both computational and experimental studies indicate that thousands of human protein-coding genes are directly regulated by miRNAs (Lewis et al. 2005; Lim et al. 2005; Miranda et al. 2006). Thus, miRNAs function as master regulators for many important biological processes, such as cell growth, differentiation, apoptosis, viral infection, and cancer development (Ambros 2004; Miska 2005; Calin and Croce 2006; Kent and Mendell 2006; Johnson et al. 2007).

\footnotetext{
${ }^{3}$ These authors contributed equally to this work.

${ }^{4}$ Corresponding author

E-mail xwang@radonc.wustl.edu

Article published online ahead of print. Article and publication date are at http://www.rnajournal.org/cgi/doi/10.1261/rna.039677.113.
}

Given the importance of miRNA-mediated gene regulation in disease biology, the diagnostic and therapeutic potential of miRNAs has been extensively explored in recent years. miRNAs are especially important in cancer development, because multiple miRNAs have been shown to be tumor suppressors or oncogenes (Croce 2009). To date, many studies have been published on using miRNAs as diagnostic and prognostic cancer biomarkers. In addition, clinical studies are under way to manipulate the expression level of miRNAs for the intervention of a variety of human diseases including cancer (for review, see Wurdinger and Costa 2007; Tong and Nemunaitis 2008). For example, introduction of tumor-suppressive miRNAs in human cancers may be an effective approach to suppress tumor growth (Kota et al. 2009; Rossi 2009; Valastyan et al. 2009).

For most miRNA-based therapeutic studies, naturally processed mature miRNAs are evaluated. One potential concern is that these natural miRNAs may not have the desired efficacy for functional gene regulation in therapeutic applications. In general, miRNA-mediated target suppression is relatively moderate as compared with siRNA-mediated

(c) 2013 Jiang et al. This article is distributed exclusively by the RNA Society for the first 12 months after the full-issue publication date (see http:// rnajournal.cshlp.org/site/misc/terms.xhtml). After 12 months, it is available under a Creative Commons License (Attribution-NonCommercial 3.0 Unported), as described at http://creativecommons.org/licenses/by-nc/3.0/. 
gene knockdown. Although a single miRNA can simultaneously target many genes, only a limited number of cellular phenotypes may be observed. For example, miR-200a has a significant impact on cancer metastasis by suppressing the motility of cancer cells (Gregory et al. 2008; Park et al. 2008). However, miR-200a does not have any reported regulatory role in some other important cancer cell properties such as proliferation or cell death.

The major goal of our study was to engineer new potent small RNAs for RNAi-based therapy. To this end, we proposed to improve the efficacy of disease-targeting miRNA by rational design of artificial interference RNA (termed "aiRNA" in our study). These aiRNA molecules are miRNA-siRNA chimeras, which were designed in silico based on natural miRNA as well as siRNA sequences. We hypothesized that, by simultaneously targeting genes from multiple functional categories that are related to the same disease, the therapeutic efficacy of an aiRNA can be greatly enhanced as compared with a single miRNA or siRNA. Specifically, we used human cancer as an example to test this new approach and showed that an aiRNA design based on miR-200a and AKT1 siRNA had enhanced functional efficacy by simultaneously suppressing both the motility and proliferation of cancer cells.

\section{RESULTS}

\section{aiRNAs and miRNAs share similar targeting characteristics}

Our definition for "artificial interference RNA" (aiRNA) is as follows: an RNA molecule that is not present in nature and has a similar sequence length to naturally processed mature miRNAs. By this definition, synthetic siRNAs can also be considered as a special form of aiRNA. An siRNA is typically a chemically synthesized 21-mer RNA duplex, with one strand having $100 \%$ sequence complementarity to a single intended mRNA target. siRNAs are widely used to knock down the expression of their gene targets. Thus, the analysis of siRNAs will give us useful clues about the general properties of aiRNAs.

It has been shown in previous studies that introduction of siRNAs into the cells can trigger widespread off-target effects. Hundreds of unintended genes can be directly silenced by siRNA overexpression (Jackson et al. 2003, 2006; Birmingham et al. 2006; Anderson et al. 2008). The seed region is defined as positions $2-8$ of a miRNA or siRNA sequence. Similar to the seed pairing requirement in miRNA-mediated gene targeting, recent studies indicate that many siRNA offtargets have siRNA seed-pairing sites in the $3^{\prime}$ UTRs. In addition, bioinformatics analysis indicates that multiple targeting determinants outside of the seed match, such as local AU content and base composition at certain sequence positions, are also shared by both miRNAs and siRNAs. Thus, in general, siRNA off-targeting is very similar to gene expression suppression by natural miRNAs. These studies suggest that
miRNAs and siRNAs enter identical or similar silencing complexes and mediate similar effects on their targets (Nielsen et al. 2007).

Given the high similarity between siRNA off-targeting and miRNA target regulation, it is reasonable to expect that an algorithm designed for miRNA target prediction should also be applicable to the prediction of genes targeted by aiRNAs (including siRNAs). To test this hypothesis, we analyzed the off-target signature of an siRNA targeting GAPDH. The siRNA off-targets were computationally predicted using MirTarget2, which is an algorithm developed in our previous study for miRNA target prediction (Wang 2008; Wang and El Naqa 2008). A recent independent study has demonstrated that MirTarget2 (data retrieved from miRDB) (Wang 2008) has superior performance over other common target prediction algorithms in comparative proteomic data analysis (Mestdagh et al. 2011). To experimentally validate MirTarget2 prediction results, the siRNA was overexpressed in HeLa cells, and microarrays were performed to identify siRNA off-targets globally at the transcriptome level. As expected, the single intended target, GAPDH, was silenced by the siRNA, with only $4 \%$ remaining mRNA level. Interestingly, among all siRNA off-targets predicted by MirTarget2, the majority were down-regulated by at least $10 \%$ (i.e., $<90 \%$ remaining expression) (Fig. 1). Moreover, more than one-fifth of all predicted off-targets were downregulated by at least $30 \%$. The level of off-target suppression is consistent with previous observations that siRNA off-targets are generally silenced at a more moderate level as compared with silencing of the intended targets (Jackson et al. 2003, 2006; Birmingham et al. 2006; Anderson et al. 2008). In contrast to the widespread down-regulation of predicted siRNA off-targets, $<5 \%$ of all genes expressed in HeLa cells were down-regulated by $30 \%$ or more (Fig. $1, P=3.5 \times 10^{-17}$ with $\chi^{2}$ test). Our data suggested that the targeting rules by

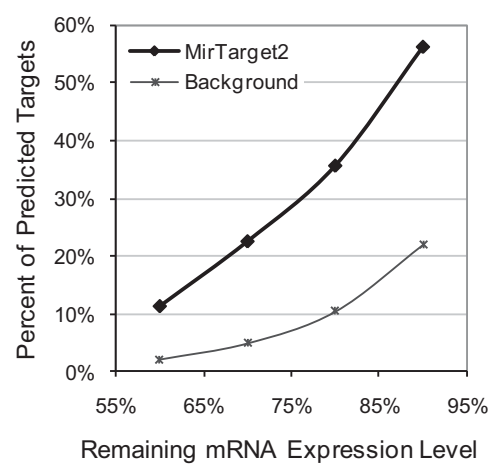

FIGURE 1. Microarrays to validate predicted siRNA off-targets. An siRNA targeting GAPDH was transfected in HeLa cells, and the transcripts down-regulated by the siRNA were globally identified with microarrays $24 \mathrm{~h}$ post-transfection. The graph shows the fraction of down-regulated off-targets among all off-targets predicted by MirTarget2 at various gene knockdown levels (represented by remaining RNA expression level). Gene down-regulation was determined by referencing to the negative control transfections. 
aiRNAs (exemplified by a GAPDH siRNA) and natural miRNAs are similar, which opens a door to design aiRNAs computationally to mimic or even enhance the functionality of naturally processed miRNAs.

\section{Design of multifunctional aiRNAs by mimicking both miR-200a and AKT1 siRNA}

We and others have previously shown that miRNA target specificity is determined by multiple sequence features, among which seed sequence match is the most critical determinant. The dominant role of seed match in miRNA target prediction was demonstrated here by comparing predicted target sets of miRNAs from the miR-200 family, which is known to suppress cancer cell motility by targeting multiple genes involved in epithelial-mesenchymal transition (EMT), leading to a significantly reduced ability of cancer cells to migrate to distant sites to form metastatic lesions (Gregory et al. 2008; Park et al. 2008). There are five members in the miR-200 family. Among them, miR-200a and miR-141 share the same seed sequence, while miR-200b, miR-200c, and miR-429 share a different seed sequence (Fig. 2A). For any pair of miR-200 members, the predicted target sets were almost identical if the seed sequence is shared by both miRNAs (miR-200a/miR-141, miR-200b/miR-200c, or miR-200b/miR-429, as shown in Fig. 2B). In contrast, there was little overlap between predicted target sets if two miRNAs have distinct seed sequences (even resulting from a single base variation). Interestingly, differences in the $3^{\prime}$ portion of the miRNA sequence were much less important than the seed region in target prediction. For example,

A

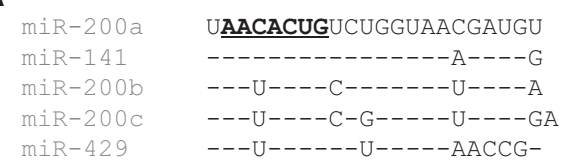

B
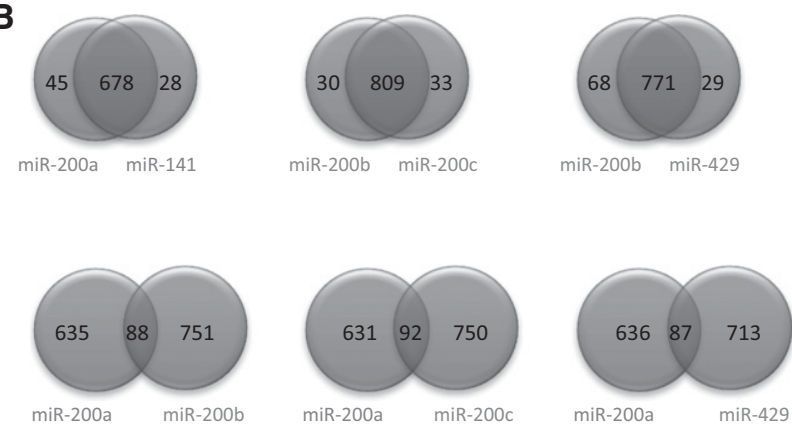

FIGURE 2. Computational prediction of gene targets of the miR-200 family. (A) Sequences of five miRNAs in the miR-200 family. The miRNA sequences were aligned to miR-200a, and identical bases to miR-200a are represented by hyphens. The seed region of miR-200a is underlined. (B) Pairwise comparison of gene sets targeted by different miR-200 family members, as predicted by MirTarget2.
miR-200b and miR-429 sequences are different by eight bases, yet the predicted targets sets for the two miRNAs were still highly similar (with $>90 \%$ overlap) because both miRNAs share the same seed region.

Given the utmost importance of seed sequence in determining miRNA targets, we hypothesized that aiRNAs sharing the same seed sequence with miR-200a should also target a very similar set of genes, leading to functional suppression of tumor cell motility. These miR-200a-mimicking aiRNAs are termed "aiR-200a" in our study. Furthermore, enhanced tumor-suppressive functions could be achieved by rational design of the non-seed sequence of aiR-200a. Specifically, our design goal was to engineer novel aiRNAs that not only share miR-200a functions for suppressing tumor cell motility, but also bear new functions for suppressing tumor cell proliferation. By simultaneously suppressing both the motility and proliferation of tumor cells, aiR-200a was expected to have enhanced functions for tumor suppression as compared with naturally processed miR-200a.

The anti-proliferation function of aiR-200a was engineered by targeting a well-established oncogene, AKT1. AKT1 is not a predicted target of any miR-200 family member. AKT1 promotes the proliferation rate of cancer cells, and overexpression of AKT1 has been observed in many human cancers (Bellacosa et al. 2005). By silencing AKT1, tumor growth can be greatly suppressed. Thus, AKT1 has been explored as an anti-cancer therapeutic target in many studies (for review, see Bellacosa et al. 2005).

In our design algorithm for aiR-200a, the siRNA targeting mechanism was invoked to knock down AKT1. The most straightforward design would have been the selection of a potent AKT1 siRNA that shares the same seed sequence with miR-200a. However, no such siRNA could be designed because the AKT1 transcript sequence does not contain any miR-200a seed binding site. Thus, an alternative strategy was employed to mimic AKT1 siRNA. Interestingly, previous studies showed that siRNAs containing a few mismatched bases to the target binding sites could still potentially knock down their intended gene targets, similarly to perfectly matched siRNAs (Jackson et al. 2003, 2006). Thus, AKT1-targeting aiR-200a could potentially be designed by introducing a few mismatched bases into an AKT1 siRNA. In this way, these aiRNAs may still silence AKT1 via RNAi-mediated transcript degradation despite the presence of base mismatches.

As the first step of aiR-200a design, we selected siRNAs to target AKT1. Design of AKT1-targeting siRNAs was performed using our recently developed tool, siOligo, which implemented a machine learning algorithm for selection of highly potent siRNAs (Wang et al. 2009). siOligo has been extensively validated experimentally for the design of hyperfunctional siRNAs, and it has been used for the design of most siRNA products by Ambion/Applied Biosystems. With siOligo, more than 1000 candidate siRNAs targeting AKT1 were evaluated, and 100 top-ranking siRNAs were selected based on their predicted knockdown efficiency. From these 
selected siRNAs, we then designed candidate aiR-200a sequences by replacing the seed region of the siRNA sequence with the miR-200a seed sequence. aiR-200a sequences with the fewest number of mismatched bases to the corresponding siRNAs were retained. In this way, three aiR-200a RNAs were designed, with two to three mismatches in the seed region to the corresponding AKT1 siRNAs (Fig. 3A). Specifically, one aiR-200a RNA has two interspersed mismatches, while the other two have two and three contiguous mismatches, respectively.

By sharing the same seed region, aiR-200a RNAs and miR200a were expected to target similar gene sets. To confirm this bioinformatically, MirTarget2 was employed for the prediction of genes targeted by miR-200a and aiR-200a, respec-

A

$$
\begin{array}{ll}
\text { miR-200a } & \text { UAACACUGUCUGGUAACGAUGU } \\
\text { aiR-200a-1 } & \text { UAACACUGCCCUACGUGAAUC } \\
\text { aiR-200a-2 } & \text { UAACACUGUUAAACCUUGCUC } \\
\text { aiR-200a-3 } & \text { AACACUGCUCCUCUGUCCCA }
\end{array}
$$

B
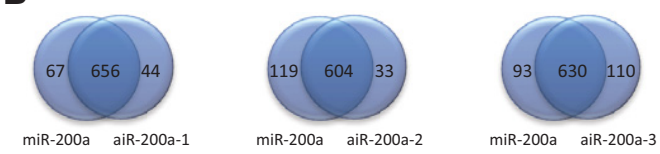

C

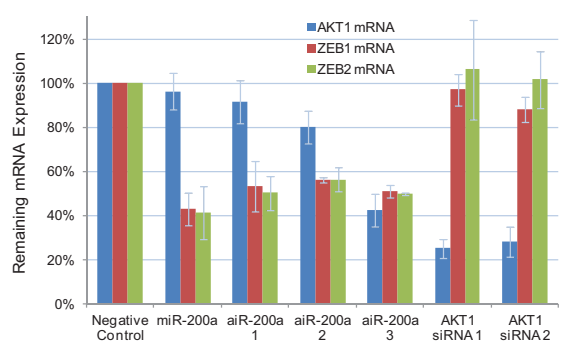

D

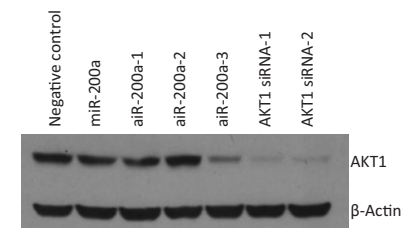

FIGURE 3. Design and validation of aiRNA-200a. (A) Sequences of three aiR-200a RNAs. These aiRNAs were designed to share the same seed sequence with miR-200a, as well as have high sequence homology to three distinct AKT1 siRNAs, respectively. The seed regions are underlined. Bases that are different from the corresponding siRNAs are highlighted in red. $(B)$ Comparison of predicted gene targets by miR-200a and aiR-200a. MirTarget2 was employed for the prediction of genes targeted by miR-200a and three aiR-200a RNAs, respectively. Each aiR-200a RNA was compared with miR-200a for predicted target overlap. $(C)$ Real-time PCR to validate predicted target suppression by miR-200a and aiR-200a. mRNA expression of three genes was assessed, including AKT1 and two previously validated targets of miR-200a, ZEB1 and ZEB2. miR-200a, three aiR-200a RNAs, and two AKT1 siRNAs were individually transfected into HeLa cells, and target gene expression was determined by comparing with the negative control transfections. $(D)$ Western blotting to evaluate suppression of AKT1 protein expression by miR-200a, aiR-200a RNAs, or AKT1 siRNAs in HeLa cells. tively, and the target sets were compared. As shown in Figure $3 \mathrm{~B}$, the vast majority of miR-200a targets were also targets of all three aiR-200a RNAs, despite the fact that the $3^{\prime}$ portions of aiR-200a and miR-200a were completely different. Specifically, 91\%, 84\%, and $87 \%$ of all predicted miR-200a targets were predicted to be targeted by the three aiRNA200a RNAs (aiRNA-200a-1, aiRNA-200a-3, and aiRNA200a-3), respectively.

\section{Experimental validation of selected aiR-200a targets}

The in silico-designed gene silencing capability of aiR-200a was evaluated experimentally. As the first step of functional validation, the suppression of three potential gene targets by aiR-200a was analyzed, including AKT1, ZEB1, and ZEB2. Among them, ZEB1 and ZEB2 are well-established miR200a targets that promote cancer metastasis (Gregory et al. 2008; Park et al. 2008), both of which were predicted to be targeted by all three aiR-200a RNAs.

First, real-time RT-PCR was performed to assess gene knockdown at the mRNA level. As shown in Figure 3C, all three aiR-200a RNAs were able to significantly suppress mRNA expression of both ZEB1 and ZEB2 by 50\%, a level comparable to that observed in miR-200a-mediated target suppression. In contrast, neither of the two AKT1 siRNAs was able to impact the expression of ZEB1 or ZEB2. On the other hand, both AKT1 siRNAs, but not miR-200a, were able to drastically silence the expression of AKT1 transcript by $>70 \%$. As to the three aiR-200a RNAs, they behaved differently in AKT1 knockdown. One of them, aiR-200a-3, was able to knock down AKT1 transcript by $\sim 60 \%$, while the other two had insignificant impact on AKT1 knockdown. The divergent capabilities of AKT1 knockdown by different aiR-200a RNAs were also confirmed at the protein expression level. As shown in Figure 3D, only aiR-200a-3, but not the other two aiRNAs, was able to suppress AKT1 protein expression to a level similar to that observed in siRNA-mediated AKT1 knockdown. Interestingly, aiR-200a-3 has two adjacent mismatched bases to the AKT1 transcript. In contrast, the other two aiRNAs have either more mismatches or interspersed mismatches (Fig. 2A). In summary, aiR-200a-3 met our design goal of silencing both AKT1 and selected miR-200a targets. Thus, this aiRNA was further evaluated for global target down-regulation at the transcriptome level.

\section{aiR-200a and miR-200a targeted similar sets of genes globally}

Global gene target regulation by aiR-200a-3 was evaluated and compared with miR-200a and AKT1 siRNAs at the transcriptome level with RNA-seq analysis. With overexpression of miR-200a, 70 genes were down-regulated by $>40 \%$, including ZEB1 and ZEB2, which were also targeted by aiRNA-200a-3 as revealed by real-time RT-PCR (Fig. 4A). Among these 70 miR-200a targets, 62 were also down-regulated by aiR-200a- 
A

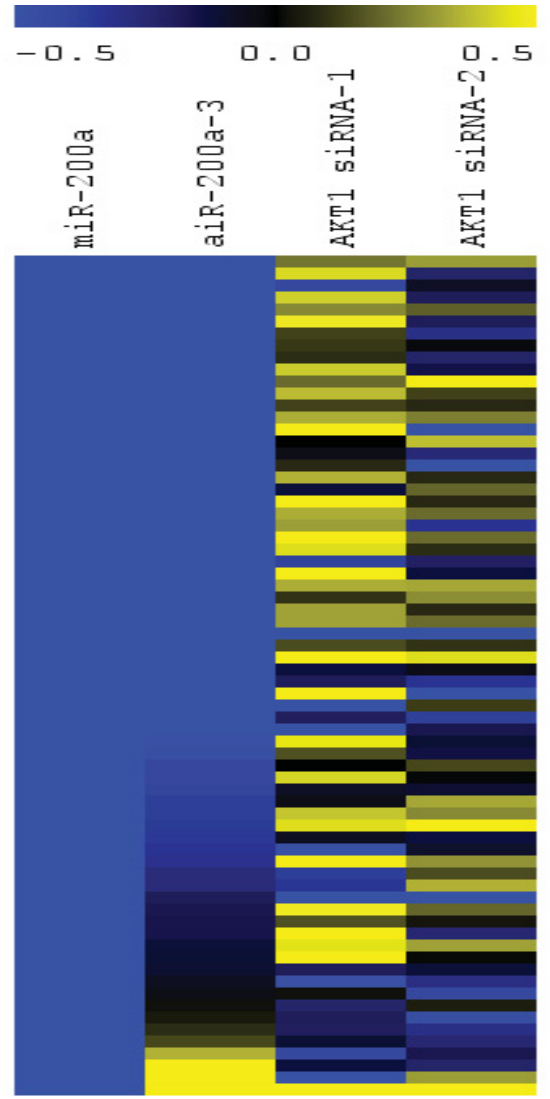

B

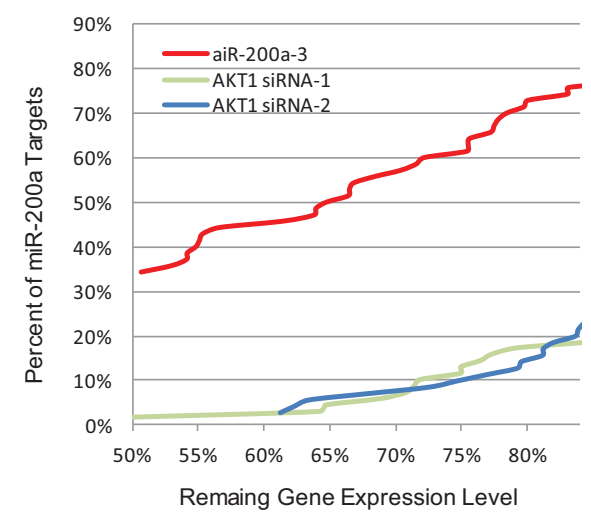

C

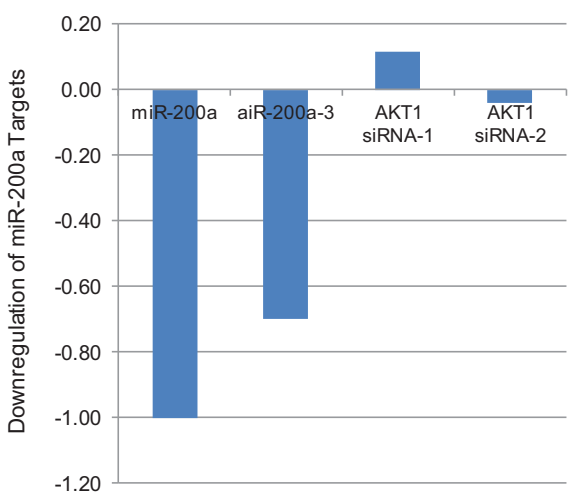

FIGURE 4. Global characterization of gene targets of aiRNA-200a with RNA-seq analysis. miR-200a, aiR-200a-3, and two AKT1 siRNAs were individually transfected into HeLa cells, and the global impact on the transcriptome was assessed with RNA-seq analysis. The sequencing data were normalized and $\log _{2}$-transformed. Gene down-regulation was determined by comparing it with the negative control transfections. In this way, 70 gene targets of miR-200a (down-regulated by at least 40\%) were identified. (A) Expression profile of 70 genes targeted by miR-200a. The expression levels of these miR-200a targets were compared under various treatment conditions (i.e., overexpression of miR-200a, aiR-200a-3, or AKT1 siRNA) in order to identify gene targets shared by miR-200a and aiR-200a-3/siRNA. (B) Percentages of miR-200a targets that were also suppressed by aiR-200a-3 or AKT1 siRNA at various remaining expression levels. $(C)$ Average level of suppression of miR-200a targets by miR-200a, aiR-200a-3, or AKT1 siRNAs.

3, including 31 targets down-regulated by $>40 \%$ (Fig. 4B; listed in Supplemental Table 1). In contrast, only a single miR200 a target $(1.4 \%$ of total) was down-regulated by $>40 \%$ by one of the two AKT1 siRNAs. Thus, most miR-200a targets were also targets of aiR-200a-3, but not AKT siRNAs. On average, these genes were suppressed to comparable levels, $50 \%$ and $38 \%$ by miR-200a and aiR-200a-3, respectively (Fig. 4C); in contrast, they were not suppressed by either of the two AKT 1 siRNAs (108\% and $97 \%$ of the negative control, respectively). In summary, both computational target prediction and RNA-seq validation data demonstrated the high similarity in global target regulation profiles by miR-200a and aiR200a-3.

\section{Multifunctional suppression of cancer cells by aiR-200a}

By targeting multiple genes involved in EMT regulation (including ZEB1 and ZEB2), miR-200a can suppress the motility of cancer cells. Similarly, by mimicking miR-200a and targeting similar sets of genes, aiR-200a was also expected to be a suppressor for cell motility. This hypothesis was tested experimentally with transwell cell migration assays. aiR-200a-3 was overexpressed in HeLa cells, and the impact on cell motility was compared with miR-200a, AKT1 siRNAs, and negative controls. As shown in Figure 5A, both aiR-200a-3 and miR200a, but not AKT1 siRNAs, were able to significantly suppress cell migration by $\sim 60 \%$. Thus, by mimicking miR200a, aiR-200a-3 inherited the function from miR-200a for suppression of cancer cell motility.

A newly gained function of aiR-200a-3 by in silico design was the ability to suppress cancer cell proliferation through targeting AKT1. To test this additional function, MTS cell proliferation assays were performed to evaluate the anti-proliferation potential of aiR-200a-3, which was capable of suppressing AKT1 expression (Fig. 3). Similar to AKT1 siRNAs, overexpression of aiR-200a-3 led to a significant reduction of cell proliferation rate by $\sim 60 \%$ (Fig. $5 \mathrm{~B}$ ). In contrast, 
A

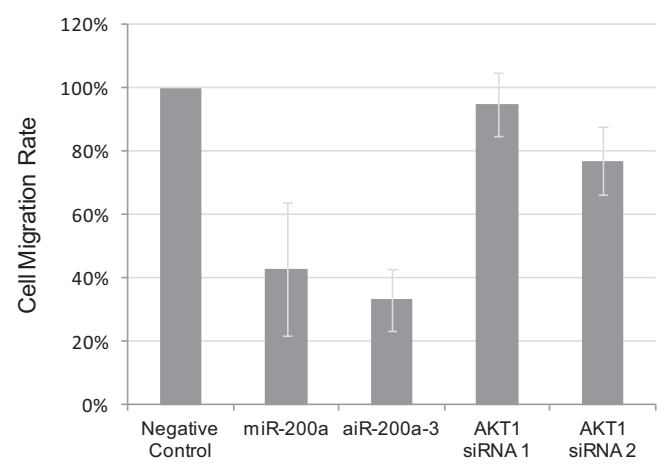

B

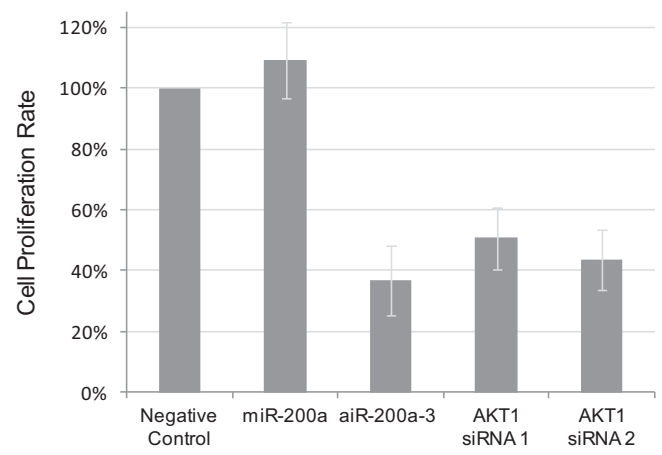

FIGURE 5. Functional characterization of aiR-200a for suppression of both the motility and proliferation of cancer cells. miR-200a, aiR-200a3, or two AKT1 siRNAs were individually transfected into HeLa cells, and changes in cell motility or proliferation were determined by comparing them with the negative control transfections. $(A)$ Transwell migration assays to assess changes in cell motility. (B) MTS assays to assess changes in cell proliferation.

overexpression of miR-200a had no impact on cell proliferation. Thus, aiR-200a-3 was able to simultaneously suppress both cancer cell migration and proliferation, whereas miR200a and AKT1 siRNA were only able to suppress either cell migration or proliferation, but not both.

\section{Additional design of multifunctional aiRNAs by mimicking both miR-9 and TP53 siRNA}

We further tested the hypothesis that the principles used for aiR-200a design can be generalized for the design of other aiRNA species by mimicking other miRNAs and siRNAs. To this end, aiR-9 aiRNAs mimicking both miR-9 and TP53 siRNAs were designed. miR-9 is capable of promoting cancer cell motility as shown in previous studies (Ma et al. 2010; Wang et al. 2011), and TP53 is a well-characterized tumor suppressor that promotes cancer cell death under chemotherapy. Thus, by mimicking both miR-9 and TP53 siRNA, aiR-9 was expected to resemble a "hyperfunctional oncogene" by promoting cancer cell motility and also avoiding cell death under chemotherapy. Although not practically useful as far as cancer cell killing is concerned, successful design of aiR-9 nevertheless would show that the general princi- ples of aiRNA design can be broadly applied to flexibly engineer new small RNAs targeting different combinations of cellular functions.

As the first step of aiR-9 design, the siOligo algorithm was used to select potent siRNAs targeting TP53 (Wang et al. 2009), as similarly described in detail for the design of aiR200a. Then, candidate aiR-9 sequences were designed by replacing the seed region of the siRNA sequences with the miR-9 seed. In this way, three aiR-9 RNAs were designed, with one or two mismatches in the seed region to the corresponding TP53 siRNAs (Fig. 6A).

We evaluated the impact of aiR-9 on regulating the expression of TP53, as well as two previously validated miR-9 targets, ALCAM 9 (Wang et al. 2011) and FSTL1 (both are involved in regulating cell motility) (data not shown) by real-time RT-PCR. As shown in Figure 6B, all three aiR-9 RNAs, but not TP53 siRNA, were able to significantly suppress the expression of both targets of miR-9. However, only one aiR-9, aiR-9-2, was also able to effectively suppress the expression of TP53. Thus, aiR-9-2 was selected for further functional validation because it met our design goal of suppressing both TP53 and selected miR-9 targets. The impact of aiR-9 on cell motility was evaluated in HeLa cells, in which TP53 functions were abrogated by HPV-induced protein degradation (Scheffner et al. 1990). As shown in Figure 7A, both miR-9 and aiR-9-2 were able to significantly promote cell motility by $44 \%$ and $57 \%$, respectively, as compared with the negative control. Thus, as expected by design, aiR-9-2 inherited the ability from miR-9 for promoting cell mobility. On the other hand, aiR-9 was also able to resemble the phenotype of TP53 knockout (TP53 ${ }^{--}$) in HCT116 cells, because both

A $\begin{array}{ll}\text { miR-9 } & \text { UCUUUGGUUAUCUAGCUGUAUGA } \\ \text { aiR-9-1 } & \text { UCUUUGGUAGUACGGUGAAG } \\ \text { aiR-9-2 } & \text { UCUUUGGUGGGAGAGGAGC } \\ \text { aiR-9-3 } & \text { UCUUUGGUCCCAGCUACUCCG }\end{array}$

B

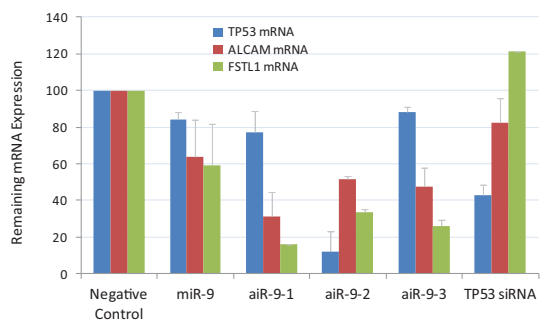

FIGURE 6. Design and validation of aiRNA-9. (A) Sequences of three aiR-9 RNAs. These aiRNAs were designed to share the same seed sequence with miR-9, as well as have high sequence homology to three distinct TP53 siRNAs, respectively. The seed regions are underlined. Bases that are different from the corresponding siRNAs are highlighted in red. (B) Real-time PCR to validate predicted target suppression by aiR-9. mRNA expression of three genes was assessed, including TP53 and two previously validated targets of miR-9, ALCAM and FSTL1. miR9, three aiR-9 RNAs, and TP53 siRNA were individually transfected into HeLa cells, and target gene expression was determined by comparing it with the negative control transfections. 
A
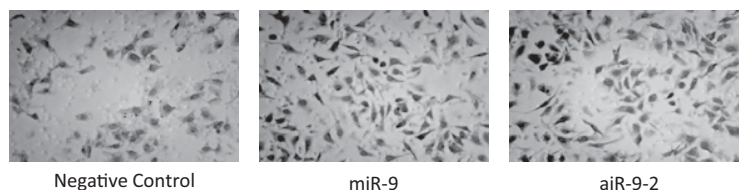

B
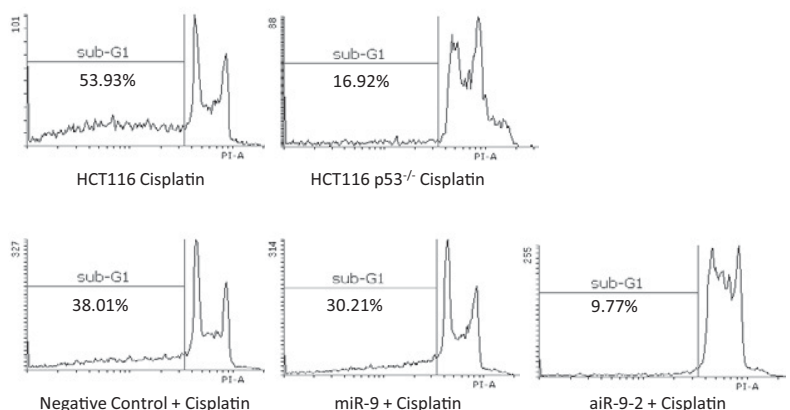

FIGURE 7. Functional characterization of aiR-9 on cell motility and cell death. (A) Impact of aiR-9 on cell motility. miR-9, aiR-9-2, and negative control RNA were individually transfected into HeLa cells, and changes in cell motility were determined by transwell migration assay. miR-9 and aiR-9-2 overexpression led to increased cell mobility to $144 \%$ and $157 \%$ on average, respectively, as compared with the control. One representative microscope image from each assay is presented here. (B) Impact of aiR-9 on cell death. TP53 deficiency renders HCT116 cells resistance to cisplatin-induced cell death. HCT116 cells and HCT116 p53 $3^{-1-}$ cells were treated with cisplatin $(50 \mu \mathrm{M})$, and cell death was determined as sub- $\mathrm{G}_{1}$ population by flow cytometry analysis after DNA PI staining. Furthermore, HCT116 cells were transfected with negative control RNA, aiR-9-2, or miR-9 individually followed by cisplatin treatment, and then analyzed by flow cytometry for cell death with PI staining.

aiR-9 overexpression and TP53 knockout led to drastic suppression of cisplatin-induced cell death as evaluated by flow cytometry analysis of sub- $\mathrm{G}_{1}$ cell population (Fig. 7B).

\section{DISCUSSION}

Because a single miRNA can regulate a large number of gene targets, miRNAs can potentially function as master switches to regulate a large portion of gene regulatory networks. The ability of multitargeting by miRNAs is important to simultaneously regulate multiple potentially redundant biological pathways, leading to observable cellular phenotypes. Thus, miRNAs have been intensely studied as potential therapeutic agents to treat a variety of human diseases. Based on the clinical importance of miRNAs, we further proposed to design aiRNAs as an improved strategy for disease treatment. Our approach may potentially alleviate the dependency on a limited number of natural miRNAs by rationally designing and testing a large number of aiRNAs for improved therapeutic efficacy. In our study, we have shown that an aiRNA mimicking miR-200a not only inherited the anti-metastatic capacity of miR-200a, but also gained the new function of anti-prolif- eration by mimicking an AKT1 siRNA. Thus, the aiRNA is considered to function equivalently to a combination of miR-200a and AKT1 siRNA. The general design principles were further validated by testing aiRNAs that mimic both miR-9 and TP53 siRNA.

A major advantage of a single multifunctional aiRNA compared with multiple miRNAs or siRNAs is that there are fewer small RNA molecules that compete for entering the RNAi machinery, thereby minimizing potential toxic effects (Grimm et al. 2006; Jackson and Linsley 2010). In addition, distinct sequences from multiple combined miRNAs/ siRNAs will significantly boost undesired sequence-specific off-target effects. Furthermore, the inclusion of a single aiRNA (in contrast to multiple miRNAs/siRNAs) will greatly simplify the therapeutic delivery process, because the pharmacokinetics and pharmacodynamics of every miRNA or siRNA included in delivery have to be closely monitored. In summary, a single multifunctional aiRNA is likely to be a more effective approach involving fewer potential side effects.

Relevant to our work, recent studies have proposed alternative designs of multitarget small RNAs to suppress a pair of distinct gene targets by using two active strands in an siRNA duplex, or suppress several functionally redundant genes from the same family by targeting homologous binding sites in the transcripts (De Guire et al. 2010; Tiemann et al. 2010; Saetrom 2013). For these design strategies, the number of intended targets is limited. In contrast, we propose to use the seed sequence of natural miRNAs for aiRNA design, thus inheriting the functions of miRNAs by suppressing many intended miRNA targets. As shown by both bioinformatics and RNA-seq analyses, the target profiles of seed-sharing aiRNAs and miRNAs are highly similar. Therefore, undesired off-target effects from aiRNAs will be greatly reduced. Our aiRNA design strategy is expected to be useful for the development of new RNAi therapeutics, in which multiple genes need to be targeted simultaneously. This represents a potentially potent strategy for more effective disease control such as triggering synthetic lethality in cancer treatment. Our aiRNA design principle can be generalized to broadly target other human diseases in addition to cancer.

Previous studies indicate that an siRNA with a few mismatched bases to the target binding site may still potentially suppress the expression of its intended gene target. However, no well-defined rules have been established to correlate siRNA knockdown efficiency to the composition of mismatches in the seed region. In our limited analysis of three aiRNAs designed to target AKT1, one inherited the knockdown capacity from the corresponding siRNA, despite the presence of two contiguous mismatched bases. In contrast, the other aiRNAs were unable to knock down AKT1, due to either three contiguous mismatches or two interspersed mismatches. Thus, it seems that both the number and positions of the mismatches in the seed region can impact aiRNA knockdown efficiency in an siRNA-like fashion. A similar design 
success rate was also observed when designing aiRNAs to target TP53. To further improve design success rate, a large number of aiRNAs with various mismatch compositions would need to be analyzed experimentally in the future to elucidate the rules of mismatch tolerance, which would eventually lead to significant algorithmic improvement.

In our study, siRNAs are considered as a special form of aiRNA, and they share similar characteristics with miRNAs and other types of aiRNA for multitargeting, which is commonly known as siRNA off-target effects. Off-targeting is an intrinsic property of siRNAs, and thus its impact should be thoroughly considered in RNAi therapeutics. Our work indicated that, by modifying the seed region of an siRNA, we were able to convert unintended off-target effects into intended miRNA-like “on-target" suppression. This provides new insights into the design of potent siRNA-based therapeutics by using the multitarget nature of siRNAs. Although miRNAs and siRNAs are still two distinct therapeutic strategies at present, we envision that the boundary defining the two would be blurred in future, because it is possible to design aiRNAs that combine the advantages of both miRNAs and siRNAs for RNAi-based therapeutics.

\section{MATERIALS AND METHODS}

\section{Cell transfection}

HeLa cells or HCT116 cells were transfected with synthetic miRNAs or siRNAs (Sigma-Aldrich) and Lipofectamine 2000 (Life Technologies). Negative controls included both transfection with a negative-control small RNA (with a random sequence) and transfection with no RNA added, and the average readings from both control transfections were used as the baseline reference for evaluating the impact of miRNA or siRNA overexpression. GAPDH siRNA was used as a positive control to monitor the transfection efficiency by real-time RT-PCR. Total RNA was extracted $24 \mathrm{~h}$ post-transfection using the mirVana RNA Isolation Kit (Life Technologies).

\section{Real-time RT-PCR}

Reverse transcription was performed with the High Capacity RT kit (Life Technologies). All PCR primers were designed in our previous study, and the sequences were retrieved from PrimerBank (Wang et al. 2012). Real-time PCR was performed to determine the relative expression levels of the transcripts. The PCR running protocol was the same as previously described (Wang and Seed 2003). Both GAPDH and $\beta$-actin were used as internal controls for gene expression normalization.

\section{Western blot}

Cells were harvested and lysed in RIPA buffer (Thermo Scientific) and a protease inhibitor mixture tablet (Roche Applied Science). The lysate was then centrifuged, and the protein supernatant was applied to SDS-PAGE. Separated protein bands on the gel were transferred to Immun-Blot PVDF membrane (Bio-Rad). The membrane was first blocked with 5\% non-fat milk in Tris-buffered saline (TBS, $\mathrm{pH}$ 7.4) and then incubated with primary anti-AKT1 mAb (Cell Signaling) or anti- $\beta$-Actin $\mathrm{mAb}$ (Sigma-Aldrich). After washing with TBS, the blots were incubated with secondary anti-mouse IgG $\mathrm{mAb}$ conjugated with horseradish peroxidase (Thermo Scientific). Bound antibodies were visualized by HRP substrate (Millipore) and chemiluminescent signal was detected on X-ray films.

\section{Transwell migration assay}

Cultured cells were resuspended and washed with PBS buffer. Fifty thousand cells in $100 \mu \mathrm{L}$ of serum-free medium were added to the upper chamber of each Transwell (24-well insert with 8 - $\mu$ m pore size from Costar). The lower chamber was filled with $600 \mu \mathrm{L}$ of medium containing $10 \%$ bovine serum. Cells were incubated for $6 \mathrm{~h}$, and cells on top of the membrane were removed with a cottontipped swab. Cells that had migrated through the membrane were fixed and stained with Hema-3 (Fisher Scientific) for counting.

\section{Cell proliferation assay}

Three days after transfection, the cell proliferation rate was determined with the MTS kit (Promega). In brief, $10 \mu \mathrm{L}$ of MTS substrate was added into each well containing $100 \mu \mathrm{L}$ of $10 \%$ FBS medium in a 96-well plate. The plate was then incubated for $3 \mathrm{~h}$, and the signal was measured using a BioTek ELx800 microplate reader.

\section{Microarray}

Microarrays were performed at the Washington University Genome Center using the Illumina BeadChip platform. The RNA quality was assessed by an Agilent 2100 Bioanalyzer (Agilent Technologies). The RNA was first amplified with the MessageAmp TotalPrep kit (Life Technologies). Amplified RNA samples were then applied to BeadChip arrays according to the manufacturer's protocols for hybridization and washing. Arrays were scanned with a Illumina BeadArray Reader, and images were analyzed by Illumina Beadscan and Beadstudio software. On-slide spot replicates were averaged and reported. Raw array data were normalized using the quantile normalization method.

\section{RNA-seq}

Total RNA was used to construct cDNA libraries for high-throughput RNA sequencing. First, ribosomal RNA (rRNA) was removed using the RiboMinus kit (Life Technologies) and custom designed rRNA oligonucleotide probes, following the manufacturer's protocol. rRNA-depleted total RNA was then used as template to construct RNA-seq libraries with the NEBNext mRNA Library Prep kit (New England BioLabs). In brief, double-stranded cDNA was synthesized from rRNA-depleted total RNA, end repaired, dA tailed, and then ligated to standard Illumina adaptor oligos. Adaptor-ligated cDNA libraries were amplified using Phusion PCR master mix. Amplified cDNA libraries were loaded into HiSeq 2000 (Illumina) for sequencing at the Washington University Genome Center. The resulting raw sequence reads were preprocessed with a custom bioinformatics pipeline to remove low-quality reads and clustered before mapping to the human transcriptome and genome (version 
19) with Bowtie (Langmead et al. 2009). In this way, 90\% of all sequence reads were mapped to human sequences. Sequence reads mapped to the same transcript were combined and then normalized by the length of the transcript as well as the number of total reads from each sample (reads per kilobase per million, RPKM). Normalized read counts were compared across samples to assess changes in transcript abundance, and are listed in Supplemental Table 2. Transcripts from the same gene locus were combined for evaluation of expression changes at the gene level.

\section{Flow cytometry}

HCT116 and HCT116 p53 ${ }^{-/-}$cells were cultured in DMEM with 10\% FBS. Cisplatin, DNase-free RNase, and Propidum Iodide (PI) were purchased from Sigma-Aldrich. After treatment with cisplatin $(50 \mu \mathrm{M})$, all cells (both floating and attached) were collected, washed once with DPBS, and then fixed with $70 \%$ ethanol on ice for at least $30 \mathrm{~min}$. Fixed cells were washed twice with DPBS and then stained with PI solution $(25 \mu \mathrm{g} / \mathrm{mL}$ PI and $100 \mu \mathrm{g} / \mathrm{mL}$ DNase-free RNase diluted in PBS) for $1 \mathrm{~h}$ at room temperature avoiding light exposure. Data were acquired by BD Aris FACS and analyzed using Flowing Software 2 version 2.5.0 (Finland).

\section{DATA DEPOSITION}

The microarray data were deposited in the NCBI GEO database (accession no. GSE41924).

\section{SUPPLEMENTAL MATERIAL}

Supplemental material is available for this article.

\section{ACKNOWLEDGMENTS}

This work was supported by the National Institutes of Health (grant R01GM089784).

Received April 19, 2013; accepted September 7, 2013.

\section{REFERENCES}

Ambros V. 2004. The functions of animal microRNAs. Nature 431: $350-355$.

Anderson EM, Birmingham A, Baskerville S, Reynolds A, Maksimova E, Leake D, Fedorov Y, Karpilow J, Khvorova A. 2008. Experimental validation of the importance of seed complement frequency to siRNA specificity. RNA 14: 853-861.

Bellacosa A, Kumar CC, Di Cristofano A, Testa JR. 2005. Activation of AKT kinases in cancer: Implications for therapeutic targeting. $A d v$ Cancer Res 94: 29-86.

Birmingham A, Anderson EM, Reynolds A, Ilsley-Tyree D, Leake D, Fedorov Y, Baskerville S, Maksimova E, Robinson K, Karpilow J, et al. 2006. 3' UTR seed matches, but not overall identity, are associated with RNAi off-targets. Nat Methods 3: 199-204.

Calin GA, Croce CM. 2006. MicroRNA signatures in human cancers. Nat Rev Cancer 6: 857-866.

Croce CM. 2009. Causes and consequences of microRNA dysregulation in cancer. Nat Rev Genet 10: 704-714.

De Guire V, Caron M, Scott N, Menard C, Gaumont-Leclerc MF, Chartrand P, Major F, Ferbeyre G. 2010. Designing small multiple-target artificial RNAs. Nucleic Acids Res 38: e140.
Denli AM, Hannon GJ. 2003. RNAi: An ever-growing puzzle. Trends Biochem Sci 28: 196-201.

Gregory PA, Bert AG, Paterson EL, Barry SC, Tsykin A, Farshid G, Vadas MA, Khew-Goodall Y, Goodall GJ. 2008. The miR-200 family and miR-205 regulate epithelial to mesenchymal transition by targeting ZEB1 and SIP1. Nat Cell Biol 10: 593-601.

Grimm D, Streetz KL, Jopling CL, Storm TA, Pandey K, Davis CR, Marion P, Salazar F, Kay MA. 2006. Fatality in mice due to oversaturation of cellular microRNA/short hairpin RNA pathways. Nature 441: $537-541$.

Hannon GJ. 2002. RNA interference. Nature 418: 244-251.

Jackson AL, Linsley PS. 2010. Recognizing and avoiding siRNA off-target effects for target identification and therapeutic application. Nat Rev Drug Discov 9: 57-67.

Jackson AL, Bartz SR, Schelter J, Kobayashi SV, Burchard J, Mao M, Li B, Cavet G, Linsley PS. 2003. Expression profiling reveals off-target gene regulation by RNAi. Nat Biotechnol 21: 635-637.

Jackson AL, Burchard J, Leake D, Reynolds A, Schelter J, Guo J, Johnson JM, Lim L, Karpilow J, Nichols K, et al. 2006. Position-specific chemical modification of siRNAs reduces "off-target" transcript silencing. RNA 12: 1197-1205.

Johnson CD, Esquela-Kerscher A, Stefani G, Byrom M, Kelnar K, Ovcharenko D, Wilson M, Wang X, Shelton J, Shingara J, et al. 2007. The let-7 microRNA represses cell proliferation pathways in human cells. Cancer Res 67: 7713-7722.

Kent OA, Mendell JT. 2006. A small piece in the cancer puzzle: microRNAs as tumor suppressors and oncogenes. Oncogene 25: 6188-6196.

Kota J, Chivukula RR, O’Donnell KA, Wentzel EA, Montgomery CL, Hwang HW, Chang TC, Vivekanandan P, Torbenson M, Clark KR, et al. 2009. Therapeutic microRNA delivery suppresses tumorigenesis in a murine liver cancer model. Cell 137: $1005-1017$.

Kozomara A, Griffiths-Jones S. 2011. miRBase: Integrating microRNA annotation and deep-sequencing data. Nucleic Acids Res 39: D152D157.

Langmead B, Trapnell C, Pop M, Salzberg SL. 2009. Ultrafast and memory-efficient alignment of short DNA sequences to the human genome. Genome Biol 10: R25.

Lewis BP, Burge CB, Bartel DP. 2005. Conserved seed pairing, often flanked by adenosines, indicates that thousands of human genes are microRNA targets. Cell 120: 15-20.

Lim LP, Lau NC, Garrett-Engele P, Grimson A, Schelter JM, Castle J, Bartel DP, Linsley PS, Johnson JM. 2005. Microarray analysis shows that some microRNAs downregulate large numbers of target mRNAs. Nature 433: 769-773.

Ma L, Young J, Prabhala H, Pan E, Mestdagh P, Muth D, TeruyaFeldstein J, Reinhardt F, Onder TT, Valastyan S, et al. 2010. miR9, a MYC/MYCN-activated microRNA, regulates E-cadherin and cancer metastasis. Nat Cell Biol 12: 247-256.

Mestdagh P, Lefever S, Pattyn F, Ridzon D, Fredlund E, Fieuw A, Ongenaert M, Vermeulen J, De Paepe A, Wong L, et al. 2011. The microRNA body map: Dissecting microRNA function through integrative genomics. Nucleic Acids Res 39: e136.

Miranda KC, Huynh T, Tay Y, Ang YS, Tam WL, Thomson AM, Lim B, Rigoutsos I. 2006. A pattern-based method for the identification of microRNA binding sites and their corresponding heteroduplexes. Cell 126: 1203-1217.

Miska EA. 2005. How microRNAs control cell division, differentiation and death. Curr Opin Genet Dev 15: 563-568.

Nielsen CB, Shomron N, Sandberg R, Hornstein E, Kitzman J, Burge CB. 2007. Determinants of targeting by endogenous and exogenous microRNAs and siRNAs. RNA 13: 1894-1910.

Park SM, Gaur AB, Lengyel E, Peter ME. 2008. The miR-200 family determines the epithelial phenotype of cancer cells by targeting the E-cadherin repressors ZEB1 and ZEB2. Genes Dev 22: 894-907.

Rossi JJ. 2009. New hope for a microRNA therapy for liver cancer. Cell 137: 990-992. 
Saetrom P. 2013. Designing dual-targeting siRNA duplexes having two active strands that combine siRNA and microRNA-like targeting. Methods Mol Biol 942: 169-177.

Scheffner M, Werness BA, Huibregtse JM, Levine AJ, Howley PM. 1990 The E6 oncoprotein encoded by human papillomavirus types 16 and 18 promotes the degradation of p53. Cell 63: 1129-1136.

Sontheimer EJ. 2005. Assembly and function of RNA silencing complexes. Nat Rev Mol Cell Biol 6: 127-138.

Tiemann K, Hohn B, Ehsani A, Forman SJ, Rossi JJ, Saetrom P. 2010. Dual-targeting siRNAs. RNA 16: 1275-1284.

Tong AW, Nemunaitis J. 2008. Modulation of miRNA activity in human cancer: A new paradigm for cancer gene therapy? Cancer Gene Ther 15: 341-355.

Valastyan S, Reinhardt F, Benaich N, Calogrias D, Szasz AM, Wang ZC, Brock JE, Richardson AL, Weinberg RA. 2009. A pleiotropically acting microRNA, miR-31, inhibits breast cancer metastasis. Cell 137: $1032-1046$.

Wang X. 2008. miRDB: A microRNA target prediction and functional annotation database with a wiki interface. RNA 14: 1012-1017.
Wang X, El Naqa IM. 2008. Prediction of both conserved and nonconserved microRNA targets in animals. Bioinformatics 24: 325-332.

Wang X, Seed B. 2003. A PCR primer bank for quantitative gene expression analysis. Nucleic Acids Res 31: e154.

Wang X, Wang X, Varma RK, Beauchamp L, Magdaleno S, Sendera TJ. 2009. Selection of hyperfunctional siRNAs with improved potency and specificity. Nucleic Acids Res 37: e152.

Wang J, Gu Z, Ni P, Qiao Y, Chen C, Liu X, Lin J, Chen N, Fan Q. 2011. NF- $\kappa$ B P50/P65 hetero-dimer mediates differential regulation of CD166/ALCAM expression via interaction with micoRNA-9 after serum deprivation, providing evidence for a novel negative auto-regulatory loop. Nucleic Acids Res 39: 64406455.

Wang X, Spandidos A, Wang H, Seed B. 2012. PrimerBank: A PCR primer database for quantitative gene expression analysis, 2012 update. Nucleic Acids Res 40: D1144-D1149.

Wurdinger T, Costa FF. 2007. Molecular therapy in the microRNA era. Pharmacogenomics J 7: 297-304. 

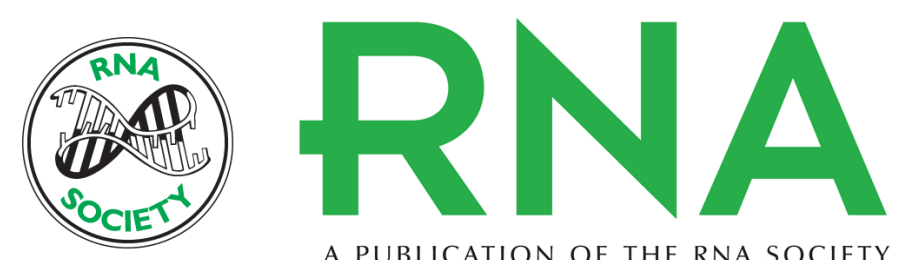

A PUBLICATION OF THE RNA SOCIETY

\section{Rational design of microRNA-siRNA chimeras for multifunctional target suppression}

Zhou Jiang, Weijun Liu, Yuhui Wang, et al.

RNA 2013 19: 1745-1754 originally published online October 21, 2013

Access the most recent version at doi:10.1261/rna.039677.113

\section{Supplemental http://rnajournal.cshlp.org/content/suppl/2013/09/24/rna.039677.113.DC1 Material}

References This article cites 41 articles, 7 of which can be accessed free at: http://rnajournal.cshlp.org/content/19/12/1745.full.html\#ref-list-1

Creative This article is distributed exclusively by the RNA Society for the first 12 months after the Commons License full-issue publication date (see http://rnajournal.cshlp.org/site/misc/terms.xhtml). After 12 months, it is available under a Creative Commons License (Attribution-NonCommercial 3.0 Unported), as described at http://creativecommons.org/licenses/by-nc/3.0/.
Email Alerting Receive free email alerts when new articles cite this article - sign up in the box at the Service top right corner of the article or click here.

\section{|||||||| Providing Precise Solutions for your research.}

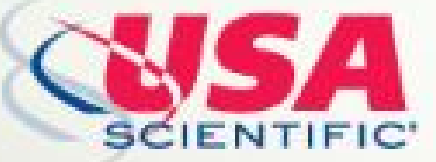

To subscribe to $R N A$ go to:

http://rnajournal.cshlp.org/subscriptions

(C) 2013 Jiang et al.; Published by Cold Spring Harbor Laboratory Press for the RNA Society 